\title{
Alveolar rhabdomyosarcoma of the sphenoid sinus mimicking optic neuritis presenting with intermittent visual loss in an adult
}

This article was published in the following Dove Press journal:

OncoTargets and Therapy

14 October 2016

Number of times this article has been viewed

\author{
Wendong Liu' \\ Libin Jiang' \\ Yulan Jin² \\ Bentao Yang ${ }^{3}$ \\ Timothy YY Lai ${ }^{4}$ \\ 'Beijing Tongren Eye Center, Beijing \\ Tongren Hospital, Capital Medical \\ University, Beijing Ophthalmology \\ and Visual Sciences Key Laboratory, \\ ${ }^{2}$ Department of Pathology, \\ ${ }^{3}$ Department of Radiology, Beijing \\ Tongren Hospital, Capital Medical \\ University, Beijing, ${ }^{4}$ Department of \\ Ophthalmology \& Visual Sciences, \\ The Chinese University of Hong \\ Kong, Shatin, Hong Kong, People's \\ Republic of China
}

\begin{abstract}
A 23-year-old male with intermittent visual loss in his left eye for 4 months was originally treated as optic neuritis elsewhere. Upon presentation to our hospital (Beijing Tongren Hospital), his left eye visual acuity was reduced to hand motion at face. The left eye ocular motility was limited in upgaze, downgaze, adduction, and abduction. Computed tomography and magnetic resonance imaging revealed lesions in the sphenoid and ethmoid sinuses, orbital apex and carvernous sinus invading adjacent extraocular muscles. Endoscopic ethmo-sphenoidostomy and debulking surgery was performed and histopathology of the specimen demonstrated alveolar rhabdomyosarcoma. Despite having treatment with a combination of orbital radiation therapy and systemic chemotherapy, the patient developed generalized metastasis with cachexia and the left eye became no light perception due to optic atrophy.
\end{abstract}

Keywords: alveolar rhabdomyosarcoma, sphenoid sinus, optic neuritis, misdiagnosis

\section{Introduction}

Rhabdomyosarcoma (RMS), a rare malignancy originating from striated muscle tissue or primitive mesenchymal tissue, is the most common soft tissue sarcoma in the pediatric population. Only a very small portion of RMS cases have been reported to develop in the adult population older than 20 years. RMS accounts for $\sim 50 \%$ of all malignant soft tissue tumors in the pediatric population and $45 \%$ of these occur in head and neck region. Adult RMS accounts for only $1 \%-3 \%$ of all malignant soft tissue tumors. ${ }^{1,2}$ As symptoms and signs of RMS result from tumor expansion into surrounding structures, clinical presentation varies according to the primary site. The early symptom of paranasal sinus RMS is atypical due to the relatively concealed location and it commonly manifests with nasal obstruction, swelling of the maxillary region or headache, purulent nasal discharge or epistaxis. ${ }^{3,4}$ Due to the lack of typically clinical manifestation, RMS is frequently misdiagnosed as an inflammatory disease. We report a 23 -year-old male who presented with a course of intermittent visual loss mimicking optic neuritis caused by alveolar RMS (ARMS) originating from the sphenoid sinuses. Based on the current literature, cases of ARMS of the sphenoid sinus have rarely been reported, and the reported patients were children or mostly presented with proptosis, epiphora, or orbital apex syndrome. We hereby report an adult ARMS of the sphenoid sinus manifesting as optic neuritis, which has not been previously reported.

\section{Case report}

A 23-year-old male with a right artificial eye due to previous ocular trauma presented to our hospital complaining of episodes of left eye progressive visual loss without any 
peri- or retro-orbital pain over 4 months. He was previously diagnosed as having visual loss due to optic neuritis elsewhere and was treated with large doses of intravenous methylprednisolone (250 to $1,000 \mathrm{mg}$ per day for four episodes) and oral prednisone $(1 \mathrm{mg} / \mathrm{kg}$ ) by local hospital on several occasions. According to the patient, no neurological imaging had been performed previously. The best corrected visual acuity (BCVA) of left eye at the disease onset was counting fingers at 3 feet. During the 4 months of corticosteroid treatment, his left eye visual acuity fluctuated considerably, with the best BCVA reaching 20/20. Upon transfer to our hospital, the BCVA of the left eye had decreased to hand motion at face with limited ocular motility in upgaze, downgaze, adduction, and abduction. The left eye pupillary reflex was sluggish. Intraocular pressure and anterior segment examination were normal, and dilated fundus examination demonstrated pallor of the left optic disc. His right eye was an artificial eye due to ocular trauma 10 years ago. He had no significant past history or family history associated with ocular disease or tumors.

Inflammatory markers tested including white blood cell count, C-reactive protein, and erythrocyte sedimentation rate were all within the normal range. He was also found to have hyperglycemia with fasting blood glucose of $9.6 \mathrm{mmol} / \mathrm{L}$ and HbA1c of $9.1 \%$ due to possible long-term use of systemic corticosteroids. On computed tomography, the left ethmoid and sphenoid sinuses were filled with an isodense mass compared with the extraocular muscles with bone destruction (Figure 1). Further assessment with magnetic resonance imaging showed a mass with homogeneous isointensity originating from the left ethmoid sphenoid sinuses and extending to the cavernous sinus and orbital apex with significant enhancement (Figure 2). The patient was subsequently transferred to otorhinolaryngological (ear, nose, and throat [ENT]) department for further assessment.

Upon transfer to the ENT department, an endoscopic ethmo-sphenoidostomy and debulking surgery were performed under general anesthesia. Frozen section analysis showed multiple small round cells consistent with a malignant neoplasm. Histopathology of the surgical specimens demonstrated a malignant neoplasm consisting of small round cells. Immunohistochemical analysis by the labelled streptavidin biotin showed diffusely nuclear-positive for myogenin (Clone F5D, MAB-0362, Fuzhou Maixin Biotechnology Development Co., Ltd., Fuzhou, People's Republic of China), cytomembraneand cytoplasmic-positive for vimentin (Clone V9, Kit-0019, Fuzhou Maixin Biotechnology Development Co., Ltd.), and cytoplasmic-positive for desmin (Clone D33, Kit-0023, Fuzhou Maixin Biotechnology Development Co., Ltd.) confirming a diagnosis of ARMS (Figure 3). Position emission tomography-computed tomography was performed to detect for distant metastasis and the result showed hypermetabolism in the liver, indicating a stage IV RMS based on the Intergroup Rhabdomyosarcoma Study TNM Pretreatment Staging System. ${ }^{5}$ The patient was immediately started on a combination of orbital radiation therapy (RT) and systemic chemotherapy.

After 3 months of treatment, the patient experienced a slight recovery of vision with the left eye BCVA improved to counting fingers at 3 feet. His ocular motility also improved slightly and the left pupil became minimally reactive. The left optic disc, however, remained pale with retinal nerve fiber layer defect. After 7 months of follow-up, his left eye visual acuity was stable. Unfortunately, at the ninth month follow-up, the left eye became no light perception after three sessions of RT due to optic atrophy associated with general metastasis with cachexia. Written consent was provided by the patient to have their data published in this case report.

\section{Discussion}

RMS is the most common sarcoma in the pediatric population, and it is very uncommon in adults. ${ }^{1,2,6,7}$ There are four histological subtypes of RMS that include pleomorphic RMS, ARMS, botryoid RMS, and embryonal RMS. ${ }^{8}$ In the head and
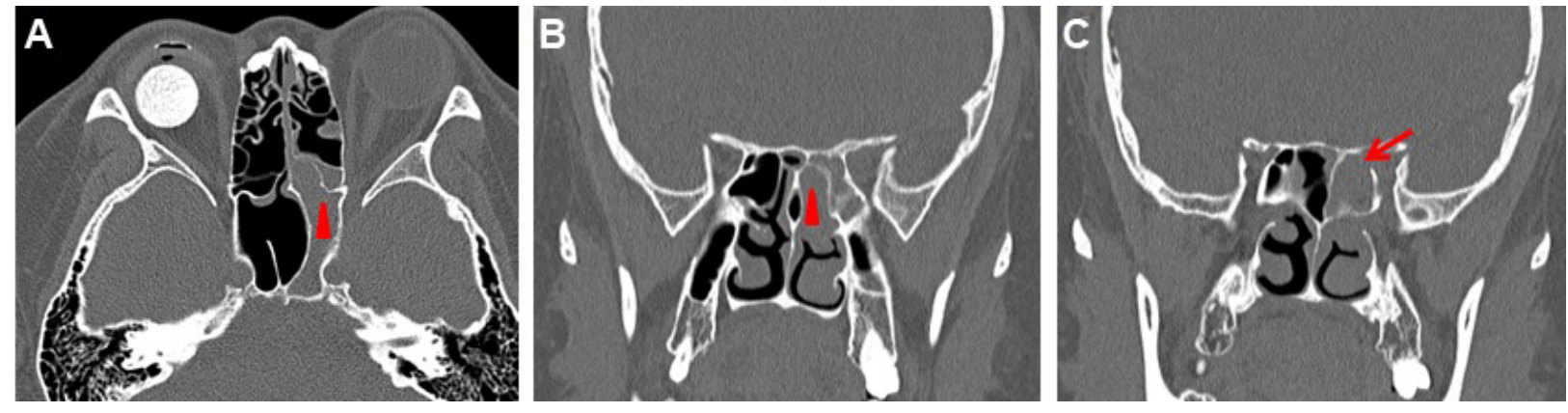

Figure I Computed tomography (CT) of the paranasal sinuses.

Notes: Axial (A) and coronal (B, C) CT scans show an isodense mass (red triangle) originating from the left ethmoid and sphenoid sinuses. Bony destruction (red arrow) is well demonstrated. 

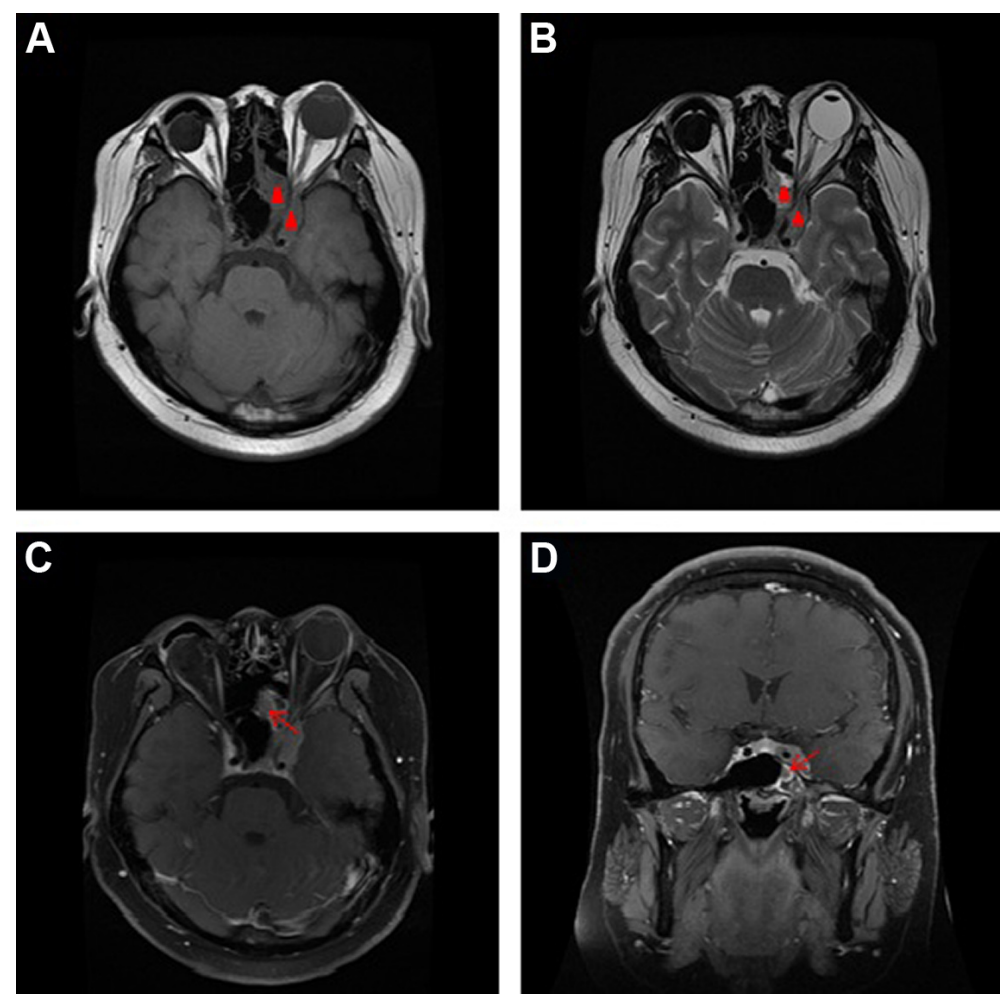

Figure 2 Magnetic resonance images of the cavernous sinus.

Notes: Axial TI weighted images (WI) (A) and T2WI (B) show the mass (red triangle) with homogeneous isointensity originating from the left ethmoid sphenoid sinuses and extending to the cavernous sinus and orbital apex. Axial (C) and coronal (D) contrast enhanced + fat suppressed TIWI show enhancement of the mass with significantly high signal intensity of marginal inflammatory secretion (red arrow).
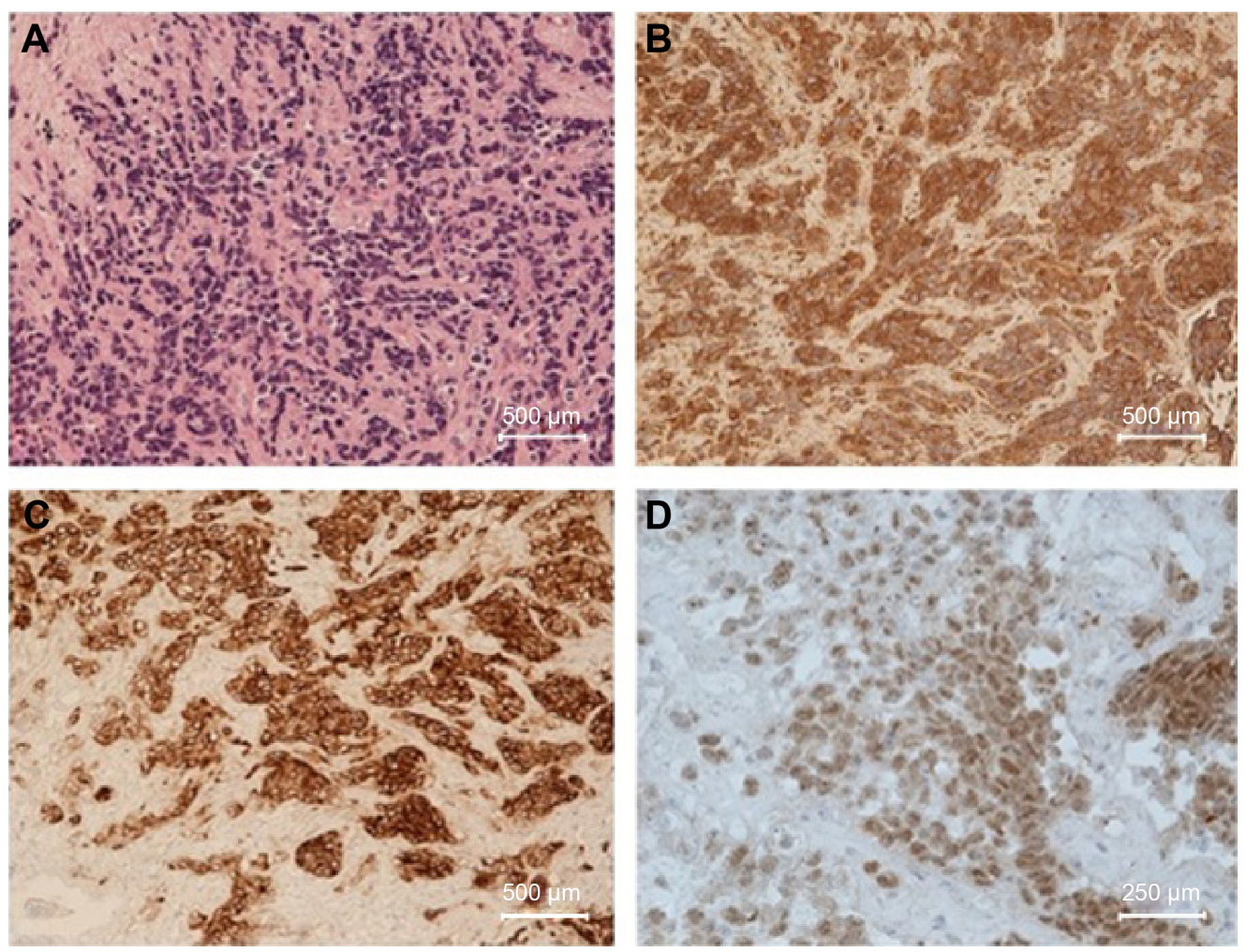

Figure 3 Photomicrographs of the surgical specimens from the left ethmoid and sphenoid sinuses.

Notes: (A) Low magnification reveals diffuse small round cells (hematoxylin and eosin, original magnification $\times 20$ ). Immunohistochemistry staining was (B) diffusely cytomembrane- and cytoplasmic-positive for vimentin (original magnification $\times 20$ ), (C) diffusely cytoplasmic-positive for desmin (original magnification $\times 20$ ), and (D) diffusely nuclear-positive for myogenin (original magnification $\times 40$ ). 
neck region, embryonal RMS is the most common variant found while the ARMS and pleomorphic RMS are rare. In recent years, the survival rate of RMS has greatly improved with the use of multimodal therapy, ${ }^{9}$ which involves surgery, chemotherapy, and RT, as proposed by the Intergroup Rhabdomyosarcoma Study group. In non-metastatic RMS, the estimated 5-year overall survival rate was $88 \%$ for embryonal RMS and $72 \%$ in ARMS.${ }^{10}$ However, the prognosis might still be poor due to the lack of early clinical symptoms of sarcomas originating in the paranasal sinuses as well as the proximity and extension into the meninges and skull base.

Our patient only presented with an intermittent and progressive visual loss with normal fundus findings initially. There was also no proptosis or abnormality in ocular movements. Moreover, the left eye visual acuity improved after using corticosteroid therapy. For these reasons, he was unfortunately misdiagnosed by the local ophthalmologists as having retrobulbar optic neuritis and was treated for several months accordingly. However, the relapse-remitting course of visual loss and limitation of ocular movements that subsequently developed suggested that the patient had diseases other than typical demyelinated optic neuritis. Neuroimaging assessments were therefore performed as soon as possible. Although the patient was treated immediately using a combination of orbital RT and systemic chemotherapy after the diagnosis of sphenoid sinus ARMS was established, he developed complete visual loss with the tumor spreading partly due to delayed treatment.

In the case presented here, there are some considerations as follows. First, the case emphasized the importance of arranging orbital or cranial neuroimaging examinations for patients with suspected visual loss related to optic nerve diseases in order to exclude orbital or neurological pathologies.
Second, the use of corticosteroid during the episode of visual loss improved the visual symptoms by alleviating tissue edema, which is caused by the tumor. However, it should not be used in a large quantity during the long-term. Finally, prompt referral of patients with sinus pathology to the ENT specialist should be carried out as tissue biopsy may be necessary for making a definite diagnosis.

\section{Disclosure}

The authors report no conflicts of interest in this work.

\section{References}

1. Wurm J, Constantinidis J, Grabenbauer GG, et al. Rhabdomyosarcomas of the nose and paranasal sinuses: treatment results in 15 cases. Otolaryngol Head Neck Surg. 2005;133(1):42-50.

2. Dumont SN, Araujo DM, Munsell MF, et al. Management and outcome of 239 adolescent and adult rhabdomyosarcoma patients. Cancer Med. 2013;2(4):553-563.

3. Makishima K, Iwasaki H, Horie A. Alveolar rhabdomyosarcoma of the ethmoid sinus. Laryngoscope. 1975;85(2):400-410.

4. Sercarz JA, Mark RJ, Tran L, Storper I, Calcaterra TC. Sarcomas of the nasal cavity and paranasal sinuses. Ann Otol Rhinol Laryngol. 1994; 103(9):699-704.

5. Crist WM, Anderson JR, Meza JL, et al. Intergroup rhabdomyosarcoma study-IV: results for patients with nonmetastatic disease. J Clin Oncol. 2001;19(12):3091-3102.

6. Shields JA, Shields CL. Rhabdomyosarcoma: review for the ophthalmologist. Surv Ophthalmol. 2003;48(1):39-57.

7. Crist W, Gehan EA, Ragab AH, et al. The third intergroup rhabdomyosarcoma study. J Clin Oncol. 1995;13(3):610-630.

8. Moon H, Kwon S, Lee J. A case of alveolar rhabdomyosarcoma of the ethmoid sinus invading the orbit in an adult. Korean J Ophthalmol. 2006; 20:70-75.

9. Raney RB, Maurer HM, Anderson JR, et al. The intergroup rhabdomyosarcoma study (IRSG): major lessons from the IRS-I through IRS-IV studies as background for the current IRS-V treatment protocols. Sarcoma. 2001;5(1):9-15.

10. Meza JL, Anderson J, Pappo AS, Meyer WH; Children's Oncology Group. Analysis of prognostic factors in patients with nonmetastatic rhabdomyosarcoma treated on intergroup rhabdomyosarcoma studies III and IV: The children's oncology group. J Clin Oncol. 2006;24(24): $3844 \mathrm{e} 51$.
OncoTargets and Therapy

\section{Publish your work in this journal}

OncoTargets and Therapy is an international, peer-reviewed, open access journal focusing on the pathological basis of all cancers, potential targets for therapy and treatment protocols employed to improve the management of cancer patients. The journal also focuses on the impact of management programs and new therapeutic agents and protocols on

\section{Dovepress}

patient perspectives such as quality of life, adherence and satisfaction. The manuscript management system is completely online and includes a very quick and fair peer-review system, which is all easy to use. Visit http://www.dovepress.com/testimonials.php to read real quotes from published authors. 